\title{
miR-1307-3p suppresses the chondrogenic differentiation of human adipose-derived stem cells by targeting BMPR2
}

\author{
ZHEN YANG ${ }^{1,2}$, RUI LI $^{2}$, JUN AO $^{3}$, QING-DE WA ${ }^{3}$, YI ZHANG $^{2}$, LONG CHEN $^{2}$,

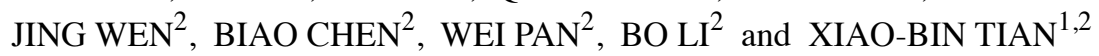 \\ ${ }^{1}$ Medical College of Guizhou University, Guiyang, Guizhou 550025; \\ ${ }^{2}$ Guizhou Provincial People's Hospital (People's Hospital of Guizhou University), Guiyang, Guizhou 550002; \\ ${ }^{3}$ Affiliated Hospital of Zunyi Medical College, Zunyi, Guizhou 563000, P.R. China
}

Received May 20, 2018; Accepted September 11, 2018

DOI: $10.3892 /$ ijmm.2018.3891

\begin{abstract}
MicroRNAs (miRs) are involved in several physiological processes, including chondrogenic differentiation, however, their expression and roles in the chondrogenic differentiation of human adipose-derived stem cells (hADSCs) remain to be fully elucidated to date. Our previous study showed that miR-1307-3p was significantly downregulated during chondrogenic differentiation by microarray and northern blot analysis. The present study aimed to investigate the effects of miR-1307-3p on chondrogenic differentiation and the underlying mechanisms. First, the decreased expression of miR-1307-3p was confirmed by reverse transcription-quantitative polymerase chain reaction analysis. Subsequently, gain- and loss-of-function of miR-1307-3p experiments showed that the overexpression of miR-1307-3p suppressed the deposition of cartilage matrix proteoglycans and decreased the expression of cartilage-related markers, including sex determining region Y-box 9, collagen type II $\alpha 1$ chain and aggrecan, whereas the knockdown of miR-1307-3p had the opposite effect. In addition, bone morphogenetic protein receptor type 2 (BMPR2) was identified as
\end{abstract}

Correspondence to: Dr Xiao-Bin Tian, Medical College of Guizhou University, 4 Beijing Road, Guiyang, Guizhou 550025, P.R. China E-mail: txb6@vip.163.com

Dr Bo Li, Guizhou Provincial People's Hospital (People's Hospital of Guizhou University), 83 Zhongshan East Road, Guiyang, Guizhou 550002, P.R. China

E-mail: libo3@medmail.com.cn

Abbreviations: BMPR2, bone morphogenetic protein receptor type 2; BMSCs, bone marrow mesenchymal stem cells; COL2A1, collagen type II $\alpha 1$ chain; hADSCs, human adipose-derived stem cells; RT-qPCR, reverse transcription-quantitative polymerase chain reaction; miRNAs, microRNAs; SOX9, sex determining region Y-box 9; TGF, transforming growth factor; 3'-UTR, 3' untranslated region

Key words: microRNA-1307-3p, human adipose-derived stem cells, chondrogenic differentiation, bone morphogenetic protein receptor type 2 , mothers against decapentaplegic homolog $1 / 5 / 8$ a target of miR-1307-3p. Further mechanistic investigations showed that miR-1307-3p attenuated the chondrogenic differentiation of hADSCs at least partly by inhibiting BMPR2-mothers against decapentaplegic signaling pathways. In conclusion, the findings revealed that miR-1307-3p inhibited the chondrogenic differentiation of hADSCs by targeting BMPR2 and its downstream signaling pathway, which may provide novel therapeutic clues for the treatment of cartilage injury.

\section{Introduction}

Cartilage tissues are degenerated and destroyed during osteoarthritis and cartilage cannot be repaired by itself. Joint replacement can efficiently relieve the pain symptoms; however, the implant can become loose and the cost is high (1). Cartilage tissue engineering has been suggested as a promising therapy for osteoarthritis (2). Among the candidates for the regeneration of articular cartilage, human adipose-derived stem cells (hADSCs) are a valuable resource. HADSCs are a population of self-renewing and multipotent cells, which are of clinical significance in cellular therapies for tissue regeneration $(3,4)$. HADSCs are capable of differentiating into several lineages, including chondrocytes $(5,6)$. As these cells can be readily harvested in abundance through a relatively non-invasive procedure, they are a valuable resource in cartilage tissue engineering. However, the regulation of the chondrogenic differentiation of hADSCs remains to be fully elucidated. Therefore, investigation of the molecular mechanism underlying the chondrogenic differentiation of hADSCs is likely to assist in the treatment of cartilage destruction.

MicroRNAs (miRNAs) are a class of small, non-coding RNAs, which are 23 nucleotides in length and usually negatively regulate target gene expression at the post-transcriptional level by incomplete complementation to nucleotides within the $3^{\prime}$ untranslated region (3'-UTR) (7). miRNAs have been found to be involved in diverse biological processes, including cell proliferation, apoptosis and differentiation (8). Increasing evidence shows that miRNAs are involved in the chondrogenic differentiation of various cell types, particularly in mesenchymal stem cells from bone marrow (BMSCs) (9). For example, miR-495 was found to be downregulated during the transforming growth factor (TGF)- $\beta 3$-induced chondrogenic 
differentiation of hMSCs using a microarray, and further functional investigations have shown that it inhibits the chondrogenic differentiation of BMSCs by directly binding and inhibiting the expression of the chondrogenic key factor sex determining region Y-box (Sox)9 (10). However, miR-410 was found to be elevated during the TGF- $\beta 3$-induced chondrogenic differentiation of MSCs, and it promoted the chondrogenic differentiation of BMSCs by negatively regulating its target gene, Wnt3a, resulting in inhibition of the Wnt signaling pathway (11). There is limited data on the involvement of miRNAs in chondrogenic differentiation hADSCs.

Zhang et al (12) analyzed the miRNA expression profile of hADSCs during chondrogenic differentiation with miRNA microarrays and found that 12 miRNAs were differentially expressed. Our previous study also analyzed the miRNA expression of hADSCs subjected to chondrogenic induction via microarray, and it was found that 20 miRNAs were significantly differently expressed. It was also identified that miR-490 promoted the chondrogenic differentiation of hADSCs by targeting bone morphogenetic protein receptor type 2 (BMPR2) (13). A study by Hou et al (14) showed that miR-193b inhibited the early chondrogenesis of hADSCs in vitro, and further mechanistic evaluation showed that it functioned by targeting TGF- $\beta$ (TGFB) 2 and TGFB receptor 3 , and inhibiting the TGF- $\beta 2$ signaling pathway. The expression of miR-92a was elevated in chondrogenic ATDC5 cells and hADSCs, and the phosphoinositide 3-kinase-Akt, ErbB and focal adhesion kinase pathways, extracellular matrix-receptor interaction, and mammalian target of rapamycin signaling pathway may be potential mediators of the effects of miR-92a on chondrogenesis (15). However, miR-194 was decreased in chondrogenic differentiation, and it suppressed chondrogenic differentiation via targeting the transcription factor Sox 5 (16). However, evidence of the role of miR-1307-3p in the chondrogenic differentiation of hADSCs remains limited.

In our previous study, profiling of the miRNA expression of hADSCs during chondrogenic differentiation was performed and the differentially expressed miRNAs verified by northern blot analysis. Furthermore, the function and mechanism of miR-490-5p in the chondrogenic differentiation of hADSCs were characterized (13). Among the differently expressed miRNAs, the expression of miR-1307-3p was significantly decreased during chondrogenic differentiation. However, whether it was involved in chondrogenic differentiation was unclear. The present study aimed to investigate the potential role of miR-1307-3p in the chondrogenic differentiation of hADSCs. The results demonstrated that miR-1307-3p suppressed the chondrogenic differentiation of hADSCs by inhibiting the BMPR2-mothers against decapentaplegic (Smad) signaling pathway via targeting BMPR2, which suggested the potential use of miR-1307-3p in hADSC-based cartilage tissue engineering.

\section{Materials and methods}

Isolation and culture of hADSCs. The hADSCs were isolated from the adipose tissue of healthy donors during liposuction at Tianjin Haihe Hospital (Tianjin, China). Written informed consent was obtained from the donors. The study was approved by the Ethics Committee of Tianjin Haihe
Hospital. Following excision during liposuction, $5 \mathrm{~g}$ of adipose tissue was minced with scissors and digested with type IA collagenase at $37^{\circ} \mathrm{C}$ for 30 min with agitation. The digested supernatants were later centrifuged at $400 \mathrm{x}$ g for $10 \mathrm{~min}$ at room temperature, and the pellet was washed with PBS and subsequently resuspended with Dulbecco's modified Eagle's medium/nutrient mixture F-12 (DMEM/F12) (Gibco; Thermo Fisher Scientific, Inc., Waltham, MA, USA). The digestion and collection procedures were repeated twice, and all of the collected cells were filtered through a 200-mesh sieve. The cells were later seeded into a culture dish and cultured in DMEM/F12 medium at $37^{\circ} \mathrm{C}$ under $5 \% \mathrm{CO}_{2}$. The medium was replaced $24 \mathrm{~h}$ later and was replaced every 3 days thereafter. The cells were passaged at 1:3 when the confluence reached $80 \%$ and cells of the third passage were used for experiments. The characterization of hADSCs was determined by flow cytometry. The corresponding antibody including rabbit anti-human antibodies for CD29 (cat. no. 12594-1-AP; 1:100), CD44 (cat. no. 15675-1-AP; 1:100), CD45 (cat. no. 20103-1-AP; 1:100), CD49 (cat. no. 21992-1-AP; 1:100), and fluorescein isothiocyanate-conjugated Affinipure Goat Anti-Rabbit IgG $(\mathrm{H}+\mathrm{L})$ (cat. no. SA00003-2; 1:100), which were purchased from ProteinTech Group, Inc. (Wuhan, China).

Induction of chondrogenic differentiation of hADSCs using a pellet culture. The induction of chondrogenic differentiation of hADSCs was performed as previously described (17). Briefly, $5 \times 10^{5}$ cells of the fourth generation were suspended in $1 \mathrm{ml}$ basal medium, which was added to $15-\mathrm{ml}$ polypropylene conical tubes and centrifuged at $500 \mathrm{x}$ g for $5 \mathrm{~min}$ at room temperature to form cell pellets. The pellets were then resuspended with $3 \mathrm{ml}$ chondrogenic medium consisting of DMEM/F-12, $5 \mathrm{ng} / \mathrm{ml}$ fibroblast growth factor- $2,10 \mathrm{ng} / \mathrm{ml}$ TGF- $\beta 1,50 \mu \mathrm{g} / \mathrm{ml}$ vitamin $\mathrm{C}$ and $10^{-7} \mathrm{M}$ dexamethasone. The cells were cultured at $37^{\circ} \mathrm{C}$ for $\sim 21$ days for differentiation and the chondrogenic medium was replaced every 2 days.

Small interfering RNA (siRNA), lentiviral vector construction and cell infection. The BMPR2-specific siRNA and control siRNA were synthesized by GenePharma (Shanghai, China). The siRNAs were transfected into cells at a final concentration of $50 \mathrm{nM}$. Recombinant lentiviruses expressing miR-1307-3p (HBLV-h-miR-1307-3p-GFP-PURO), miR-1307-3p inhibitor (HBLV-h-shmiR-1307-3p-GFP-PURO) or control lentivirus were purchased from Hanbio (Shanghai, China). Lentiviruses at a dose of $2 \times 10^{8} \mathrm{TU} / \mathrm{ml}$ and a multiplicity of infection of 10 were used to infect hADSCs cells according to the manufacturer's protocol. Following incubation with the lentivirus for $48 \mathrm{~h}$, the medium was replaced with fresh medium and the cells were cultured for further experiments. The infection efficiency was determined by microscopic examination of GFP-positive cells.

Alcian blue staining. To assess the deposition of cartilage matrix proteoglycans, the cell pellets were first fixed with $4 \%$ paraformaldehyde and dehydrated with an ethanol gradient. Subsequently, the cells were paraffin-embedded and cut into $5-\mu \mathrm{m}$ sections. The sections were first washed with PBS three times, following which they were immersed in $0.1 \mathrm{~N} \mathrm{HCL}$ for 5 min and stained with Alcian blue overnight. Following 
being washed with $0.1 \mathrm{~N}$ HCL for 5 min three times, the sections were examined using a Leica DM LB2 upright light microscope (Leica Microsystems GmbH, Wetzlar, Germany).

Immunocytochemical examination. Following the induction of chondrogenesis for 21 days, the cells pellets were fixed with $4 \%$ paraformaldehyde, dehydrated and paraffin-embedded as described above. The sections were then incubated with blocking solution [PBS/10\% goat serum (Beijing Biosynthesis Biotechnology Co., Ltd., Beijing, China)/0.1\% Triton X-100] for $30 \mathrm{~min}$, and were then incubated with monoclonal rabbit anti-human antibodies against collagen type II (ColII; cat. no. bsm-33129M; 1:200; Beijing Biosynthesis Biotechnology Co., Ltd.) for $2 \mathrm{~h}$ at room temperature and washed with PBS three times. Subsequently, the sections were incubated with HRP-conjugated secondary antibody for $1 \mathrm{~h}$ at room temperature (cat. no. SA00001-2; 1:1,000; ProteinTech Group, Inc.) and washed with PBS three times. Subsequently, the sections were incubated with DAB solution for $30 \mathrm{~min}$ at room temperature and washed with deionized water for $5 \mathrm{~min}$. Finally, the sections were stained with hematoxylin to identify the nuclei. Images were captured with a Leica DM LB2 upright light microscope.

RNA isolation and reverse transcription-quantitative polymerase chain reaction $(R T-q P C R)$ analysis. Total RNA was isolated using TRIzol ${ }^{\circledR}$ reagent (Invitrogen, Thermo Fisher Scientific, Inc., Waltham, MA, USA) according to the manufacturer's protocol. cDNA was synthesized using the PrimeScript RT reagent kit (Takara Biotechnology Co., Ltd., Dalian, China). RT-qPCR analysis was performed using the SYBR Premix Ex Taq kit (Takara Biotechnology Co., Ltd.). The PCR (20 $\mu \mathrm{l})$ included SYBR Green premix (10 $\mu \mathrm{l})$, cDNA (2 $\mu \mathrm{l})$, forward primer $(10 \mu \mathrm{M} ; 1 \mu \mathrm{l})$, reverse primer $(10 \mu \mathrm{M}$; $1 \mu \mathrm{l})$ and $\mathrm{H}_{2} \mathrm{O}(6 \mu \mathrm{l})$. The cycles were as follows: $95^{\circ} \mathrm{C}$ for $30 \mathrm{sec}$, followed by 40 cycles of $95^{\circ} \mathrm{C}$ for $5 \mathrm{sec}$, and $60^{\circ} \mathrm{C}$ for $30 \mathrm{sec}$. The primers used are listed in Table I. The results were normalized to the expression of U6 or GAPDH and were calculated with the $2^{-\Delta \Delta \mathrm{Cq}}$ method (18).

Western blot analysis. The cells were lysed with RIPA buffer, and the protein concentration was determined with the Bradford assay (Bio-Rad Laboratories, Hercules, CA, USA). An equal amount of protein $(30 \mu \mathrm{g})$ was separated by SDS-PAGE on a $10 \%$ gel and transferred onto a nitrocellulose membrane. Membranes were incubated with blocking buffer (cat. no. P0023B; Beyotime Institute of Biotechnology, Haimen, China) at room temperature for $2 \mathrm{~h}$. The membranes were incubated with primary antibodies against BMPR2 (cat. no. bs-4237R; 1:500), COL2A1 (cat. no. bs-11929R; 1:500), SOX9 (cat. no. bs-10725R; 1:500), Aggrecan (cat.no.bs-1223R; 1:1,000), Smad1 (cat. no. bs-16376R; 1:500), phosphorylated (p)Smad1 (cat. no. bs-3417R; 1:500), Smad5 (cat. no. bs-13890R; 1:1,000), pSmad5 (cat. no. bs-19918R; 1:500), Smad8 (sc-293413; 1:1,000), pSmad8 (cat. no. 9511L; 1:500) and GAPDH (cat. no. bs-10900R; 1:1,000) at room temperature for $2 \mathrm{~h}$, followed by secondary horseradish peroxidase-conjugated antibodies (cat. no. bs-0295G-HRP; 1:1,000) at room temperature for $2 \mathrm{~h}$ thereafter. Smad8 antibody was purchased from Santa Cruz Biotechnology, Inc., Dallas, TX,
USA and pSmad8 antibody was purchased from Cell Signaling Technology, Inc., Danvers, MA, USA; the other antibodies were purchased from Beijing Biosynthesis Biotechnology Co., Ltd., (Beijing, China). GAPDH was used as an internal control. The protein were visualized by enhanced chemiluminescence (Applygen Technologies Inc., Beijing, China). Densitometric analysis was performed using LabWorks 4.0 image acquisition and analysis software (UVP, LLC, Upland, CA, USA).

Luciferase reporter assay. Prediction of miR-1307-3p targets was performed using web-based bioinformatics tools including TargetScan (http://www.targetscan.org/vert_72/), and PicTar (www.pictar.org). The BMPR2 3' UTR harboring the miR-1307-3p target sequence and the seed sequence mutated type (BMPR2 3'UTR mut) were synthesized by GenePharma (Shanghai, China) and were then ligated following the luciferase ORF into the pmirGLO vector (Promega Corporation, Madison, WI, USA). The cells were seeded into 24-well plates at a confluence of $\sim 80 \%$ the day before transfection, thus ensuring that the cells were at a confluence of $80 \%$ on the day of transfection. miR-1307-3p mimics were cotransfected with BMPR2 3'UTR or BMPR2 3'UTR mut reporter. Transfection was performed using Lipofectamine ${ }^{\circledR} 2000$ reagent (Invitrogen; Thermo Fisher Scientific, Inc. Waltham, MA, USA). At $48 \mathrm{~h}$ post-transfection, the cells were lysed according to the manufacturer's protocol (Promega Corporation), and the luciferase activities were determined using the Dual-Glo luciferase reporter assay system (Promega Corporation).

Statistical analysis. The data are expressed as the mean \pm standard error of the mean. Statistical analyses were performed with SPSS statistical software version 16.0 (SPSS, Inc., Chicago, IL, USA) using Student's t-test and one-way analysis of variance with Bonferroni post hoc analysis. $\mathrm{P}<0.05$ was considered to indicate a statistically significant difference.

\section{Results}

miR-1307-3p is downregulated during chondrogenic differentiation. To determine the expression levels of miR-1307-3p during the chondrogenic differentiation of hADSCs, hADSCs were cultured in chondrogenic media using the pellet culture system for 21 days. First, the characterization of hADSCs was analyzed by flow cytometry. The results showed that the cells were positive for CD29, CD44 and CD49 and negative for CD45 (Fig. 1A). The chondrogenic differentiation of hADSCs was confirmed by Alcian blue staining and immunocytochemical examination of the chondrogenic marker, ColII. Morphological observation showed the morphology of cells was altered following induction and it consisted of polygon-shaped cells compared with those in the control group (Fig. 1B). The Alcian blue staining showed the deposition of cartilage matrix proteoglycans was promoted in hADSCs subjected to chondrogenic induction (Fig. 1C). In addition, the level of ColII was increased in hADSCs following induction (Fig. 1D). These results confirmed the chondrogenic differentiation of hADSCs exposed to the chondrogenic induction medium. Subsequently, RT-qPCR analysis was used to examine the expression of miR-1307-3p. As shown in Fig. 1E, the expression of miR-1307-3p was significantly decreased 
Table I. Primers and oligonucleotides used in the present study.

\begin{tabular}{|c|c|}
\hline Primer & Sequence $\left(5^{\prime}-3^{\prime}\right)$ \\
\hline miR-1307-3p-RT & GTCGTATCCAGTGCAGGGTCCGAGGTGCACTGGATACGACCACGACC \\
\hline U6-RT & AACGCTTCACGAATTTGCGTG \\
\hline \multirow[t]{2}{*}{ miR-1307-3p-qPCR } & Forward: TGCGGGTCCAGTTTTCCCAGGAA \\
\hline & Reverse: CCAGTGCAGGGTCCGAGGT \\
\hline \multirow[t]{2}{*}{ U6-qPCR } & Forward: GCTCGCTTCGGCAGCACA \\
\hline & Reverse: GAGGTATTCGCACCAGAGGA \\
\hline \multirow[t]{2}{*}{ BMPR2-qPCR } & Forward: TGGGAAAGAAACAAATCTGTGAGC \\
\hline & Reverse: GAGGAGGAAGAATAATCTGGATAAGGAC \\
\hline \multirow[t]{2}{*}{ Smad1-qPCR } & Forward: CACCTGCTTACCTGCСТCCTGAA \\
\hline & Reverse: GCAACCGCCTGAACATCTCCTCT \\
\hline \multirow[t]{2}{*}{ Smad2-qPCR } & Forward: ATGAATTAAATCAGAGGGTTGGAG \\
\hline & Reverse: CTTCTTGTCATTTCTACCGTGGC \\
\hline \multirow[t]{2}{*}{ Smad3-qPCR } & Forward: GTGACCACCAGATGAACCACAGCA \\
\hline & Reverse: CGTAGTAGGAGATGGAGCACCAGAAGG \\
\hline \multirow[t]{2}{*}{ Smad4-qPCR } & Forward: TCCAGCCTCCCATTTCCAATCAT \\
\hline & Reverse: CTCCAGAAGGGTCCACGTATCCA \\
\hline \multirow[t]{2}{*}{ Smad5-qPCR } & Forward: AAAACACTAGGCGACATATTGGA \\
\hline & Reverse: GGAATCTTACAGACAGTGGTGGG \\
\hline \multirow[t]{2}{*}{ Smad6-qPCR } & Forward: AGTGACTGCGAGACGGTGACCTGCTG \\
\hline & Reverse: GCGAGTACGTGACGGTTTTGAGTTCCTG \\
\hline \multirow[t]{2}{*}{ Smad7-qPCR } & Forward: TCTCAGGCATTCCTCGGAAGTCAAG \\
\hline & Reverse: CTAGTTCGCAGAGTCGGCTAAGGTGAT \\
\hline \multirow[t]{2}{*}{ Smad8-qPCR } & Forward: GAGGTGTATGCCGAGTGCGTGAG \\
\hline & Reverse: GCTGAGCGAAGAGCTGGTTGTTG \\
\hline \multirow[t]{2}{*}{ Noggin-qPCR } & Forward: GCCATGCCGAGCGAGATCAAAGGG \\
\hline & Reverse: CGACCACAGCCACATCTGTAACTTCCTCC \\
\hline \multirow[t]{2}{*}{ Chordin-qPCR } & Forward: GATGCCCTGATCCCAGTCCAGACG \\
\hline & Reverse: GCCACCACCTCACTGCTTGTCCCTAC \\
\hline \multirow[t]{2}{*}{ GAPDH-qPCR } & Forward: ATGACATCAAGAAGGTGGTGAAGCAGG \\
\hline & Reverse: GCGTCAAAGGTGGAGGAGTGGGT \\
\hline \multirow[t]{2}{*}{ miRNA-NC mimics } & Forward: UCACAACCUCCUAGAAAGAGUAGA \\
\hline & Reverse: UCUACUCUUUCUAGGAGGUUGUGA \\
\hline \multirow[t]{2}{*}{ miR-1307-3p mimics } & Forward: ACUCGGCGUGGCGUCGGUCGUG \\
\hline & Reverse: CACGACCGACGCCACGCCGAGU \\
\hline \multirow[t]{2}{*}{ BMPR2 3'UTR wt } & Forward: CTAGCAGGUUGUUGCAGUGAGCCGAGG \\
\hline & Reverse: TCGACCTCGGCTCACTGCAACAACCTG \\
\hline \multirow[t]{2}{*}{ BMPR2 3'UTR mut } & Forward: CTAGCAGGUUGUUGCAGUGACGGCTCG \\
\hline & Reverse: TCGACGAGCCGTCACTGCAACAACCTG \\
\hline \multirow[t]{2}{*}{ Si-BMPR2 } & Sense: GCAUGUUUGAUUCCUGAUGTT \\
\hline & Antisense: CGUACAAACUAAGGACUACTT \\
\hline \multirow[t]{2}{*}{ Control siRNA } & Sense: UUCUCCGAACGUGUCACGUTT \\
\hline & Antisense: ACGUGACACGUUCGGAGAATT \\
\hline
\end{tabular}

RT, reverse transcription; qPCR, quantitative polymerase chain reaction; miR, microRNA; BMPR2, bone morphogenetic protein receptor type 2; Smad, mothers against decapentaplegic; NC, negative control; 3'UTR, 3' untranslated region; wt, wild-type; mut, mutant.

following 21 days of induction, which was consistent with previous microarray and northern blot results.

miR-1307-3p inhibits chondrogenic differentiation of $h A D S C s$. To investigate the function of miR-1307-3p in the chondrogenic differentiation of hADSCs, the expression of miR-1307-3p was overexpressed or knocked down with a GFP-expressing lentivirus system. The hADSCs were infected with recombinant lentivirus expressing miR-1307-3p or miR-1307-3p inhibitor or control and were then subjected 
A
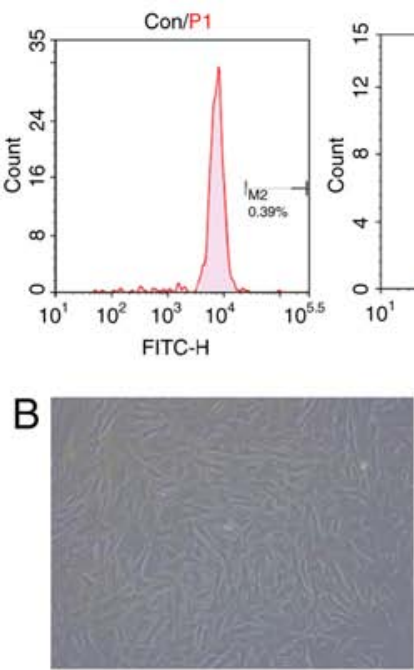

Non-induced

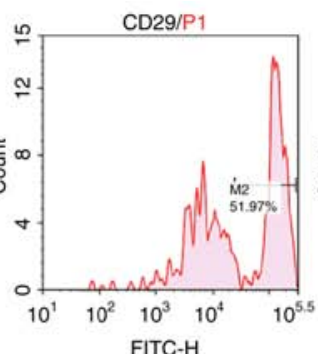

FITC-H
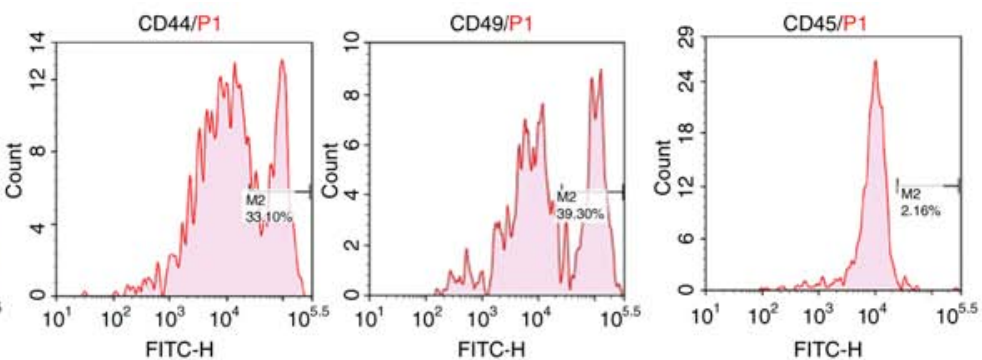

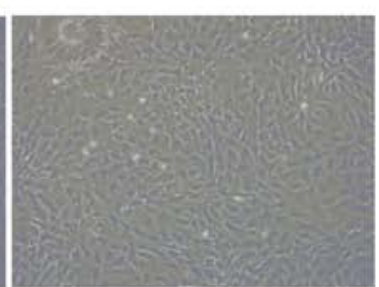

Induced

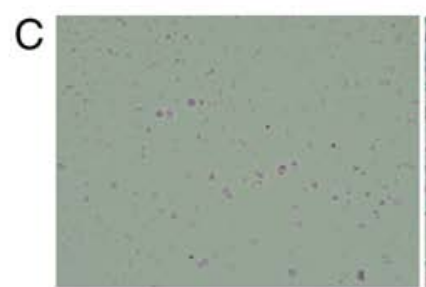

Non-induced

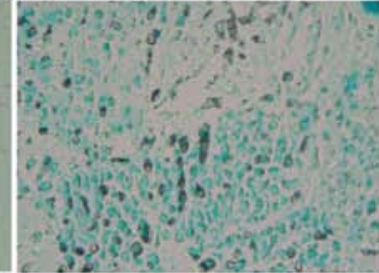

Induced

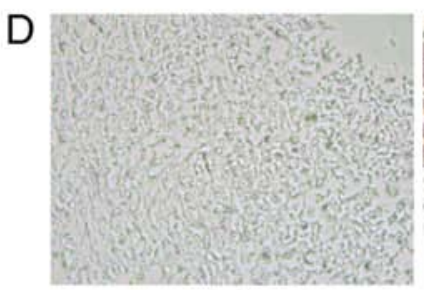

Non-induced

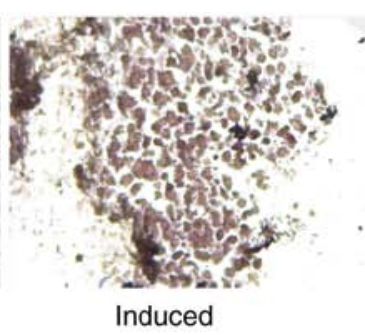

$\mathrm{E}$

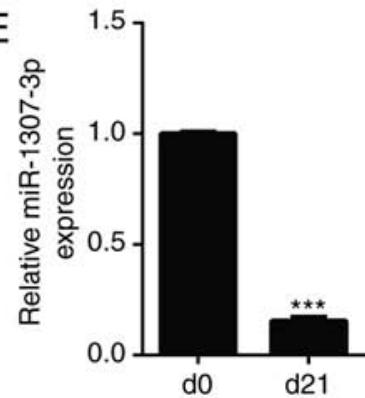

Figure 1. Expression levels of miR-1307-3p during chondrogenic differentiation of hADSCs. (A) Flow cytometric analysis of the surface markers specific for hADSCs. hADSCs were cultured using the cell pellet system and were subjected to chondrogenic differentiation for 21 days. (B) Morphology of hADSCs (magnification, x10). (C) Deposition of cartilage matrix proteoglycans was determined by Alcian blue staining (magnification, x40). (D) Expression of collagen type II was examined with immunocytochemical staining (magnification, $x 40$ ). (E) Expression of miR-1307-3p was determined by reverse transcription-quantitative polymerase chain reaction analysis. ${ }^{* * *} \mathrm{P}<0.001$. miR, microRNA; hADSCs, human adipose-derived stem cells; FITC, fluorescein isothiocyanate; d, day.

to chondrogenic differentiation. First, microscopic examination of GFP-positive cells was performed to determine the infection efficiency of the recombinant lentivirus. As shown in Fig. 2A, the infection efficiency was high as shown by the $\sim 80 \%$ GFP-positive cells in all groups. Subsequently, an RT-qPCR assay was used to determine the expression efficiency of the recombinant lentivirus. Infection with the miR-1307-3p-expressing lentivirus resulted in a significant increase of miR-1307-3p levels, whereas infection with the miR-1307-3p inhibitor lentivirus led to a decrease in its levels (Fig. 2B). The effect of miR-1307-3p on chondrogenic differentiation of hADSCs was determined by examining the deposition of cartilage matrix proteoglycans and the levels of three cartilage-related markers COL2A1, SOX9 and Aggrecan. Alcian blue staining showed the deposition of cartilage matrix proteoglycans was suppressed when miR-1307-3p was overexpressed but was promoted when miR-1307-3p was knocked down (Fig, 2C). The RT-qPCR results showed that the mRNA levels of COL2A1, SOX9 and Aggrecan were markedly decreased following miR-1307-3p overexpression, whereas they were increased in the miR-1307-3p-knockdown group (Fig. 2D). The western blot assay showed that the proteins levels of COL2A1, SOX9 and Aggrecan were significantly decreased in the miR-1307-3p-overexpressing cells, but increased following miR-1307-3p knockdown (Fig. 2E), which suggested that miR-1307-3p suppressed chondrogenic differentiation.

BMPR2 is a direct target of miR-1307-3p. To examine the underlying mechanism by which miR-1307-3p suppresses chondrogenic differentiation, potential target genes were searched for using TargetScan and PicTar. Among the predicted targets, BMPR2 was identified, which was suggestive of its involvement in chondrogenic differentiation. To confirm the involvement of BMPR2 in the chondrogenic differentiation of hADSCs, its expression during differentiation was examined. The RT-qPCR and western blot analyses showed that the mRNA and protein levels of BMPR2 were increased following 21 days of induction (Fig. 3A and B), which was in accordance with the downregulation of miR-1307-3p (Fig. 1D). Bioinformatics analysis showed that there was one putative binding site in the 3'UTR of BMPR2 mRNA (Fig. 3C). First, a dual luciferase assay was used to determine whether miR-1307-3p directly targeted BMPR2; as shown in Fig. 2D, the overexpression of miR-1307-3p significantly decreased the luciferase activity of the BMPR2 3'UTR ( 60\%) but had no effect on that of the BMPR2 3'UTR mut. Furthermore, the effect of miR-1307-3p on the endogenous levels of BMPR2 was examined. The RT-qPCR results showed that the mRNA level of BMPR 2 was decreased by $\sim 40 \%$ following miR-1307-3p 
A

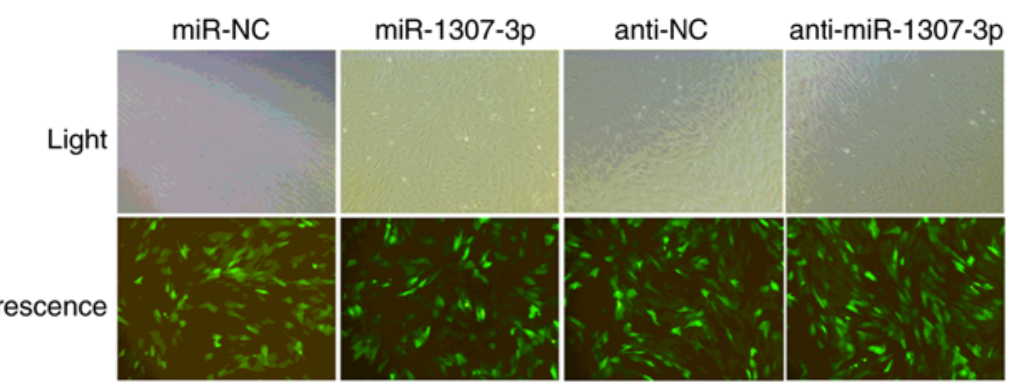

B
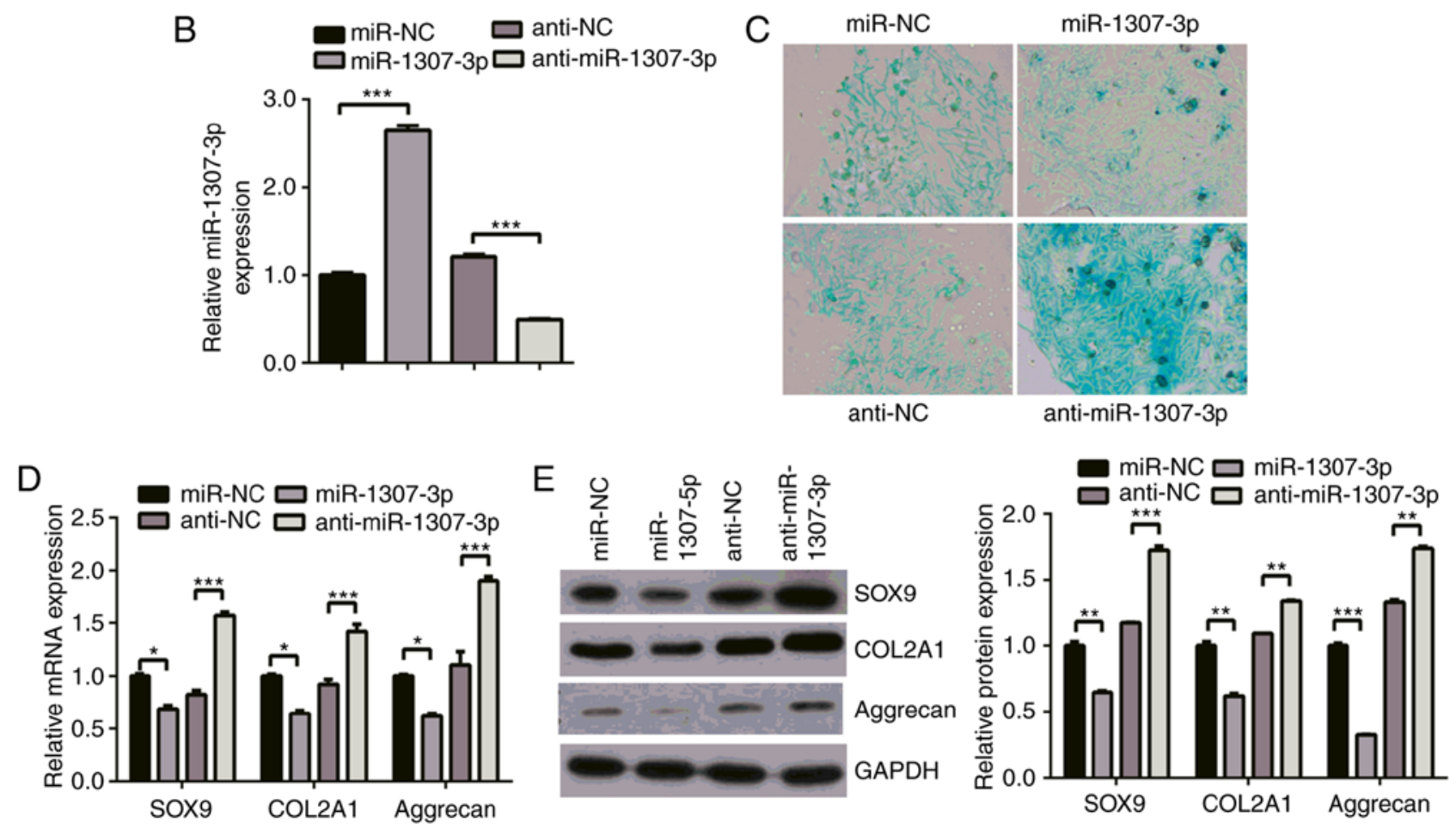

Figure 2. miR-1307-3p inhibits the chondrogenic differentiation of hADSCs. hADSCs were infected with recombinant lentivirus expressing miR-1307-3p or miR-1307-3p inhibitor or control. Following infection, chondrogenic differentiation was induced for 21 days. (A) Infection efficiency was determined by microscopic examination of the GFP-expressing cells (magnification, x10). (B) expression of miR-1307-3p was determined with reverse transcription-quantitative polymerase chain reaction analysis. (C) Deposition of cartilage matrix proteoglycans was determined by Alcian blue staining (magnification, $\mathrm{x} 40$ ). (D) mRNA levels of chondrogenic genes (SOX9, COL2A1 and aggrecan) were analyzed by RT-qPCR analysis. (E) Protein levels of SOX9, COL2A1 and aggrecan were detected with western blot analysis. ${ }^{*} \mathrm{P}<0.05,{ }^{* * *} \mathrm{P}<0.01$ and ${ }^{* * *} \mathrm{P}<0.001$. miR, microRNA; hADSCs, human adipose-derived stem cells; SOX9, sex determining region Y-box 9; COL2A1, collagen type II $\alpha 1$ chain; RT-qPCR, reverse transcription-quantitative polymerase chain reaction; NC, negative control.

overexpression, whereas the knockdown of miR-1307-3p led to the opposite results (Fig. 3E). The western blot analysis also showed that the overexpression of miR-1307-3p significantly decreased the protein levels of BMPR2, whereas miR-1307-3p knockdown increased the protein levels of BMPR2 (Fig. 3F). These results indicated that miR-1307-3p bound directly and negatively regulated the expression of BMPR2.

BMPR2 mediates the suppression of chondrogenic differentiation of hADSCs by miR-1307-3p. To determine whether BMPR2 mediated the effects of miR-1307-3p on the chondrogenic differentiation of hADSCs, si-BMPR2 was transfected into hADSCs that were infected with the miR-1307-3p inhibitor lentivirus. The RT-qPCR and western blot analyses showed that the depletion of miR-1307-3p led to an increase in the mRNA and protein levels of BMPR2, whereas the knockdown of BMPR2 attenuated the promoting effect of miR-1307 depletion on the expression of BMPR2 (Fig. 4A and B). Furthermore, the knockdown of BMPR2 prevented the promoting effect of miR-1307 inhibition on the expression of cartilage related markers COL2A1, SOX9 and Aggrecan at the mRNA and protein levels (Fig. 4C and D). These results demonstrated that BMPR2 was a functional target of miR-1307-3p.

miR-1307-3p inhibits chondrogenic differentiation via suppression of BMPR 2 downstream pathways. Considering the key role of BMPR2 in the BMPR2-Smad signaling pathway, the present study investigated whether miR-1307-3p inhibited the chondrogenic differentiation of hADSCs through suppressing the BMPR2-Smad signaling pathway. The protein levels of BMPR2 downstream molecules, including Smad1, 5 , and 8 , were examined. Western blot analysis showed that there were no significant changes in the total levels of Smad1, 5 , and 8 when miR-1307-3p was overexpressed or knocked down. However, the phosphorylation of the three Smad 

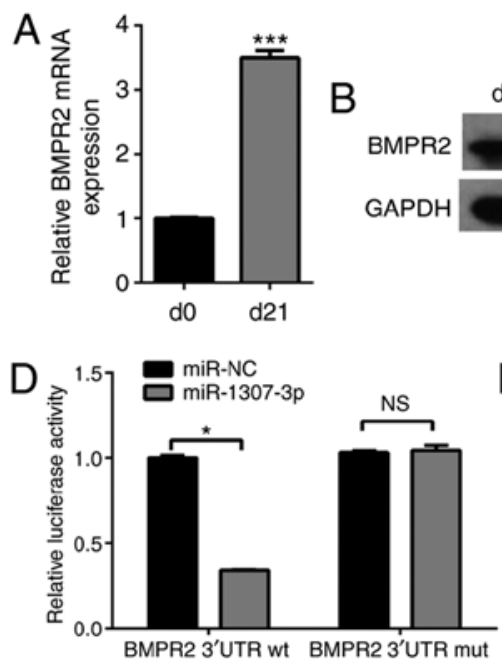

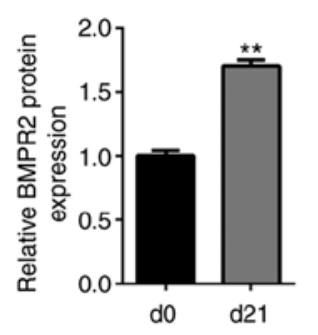

C BMPR2 3'UTR wt AGGUUGUUGCAGUGAGCCGAGA IIIIIII miR-1307-3p GUGCUGGCUGCGGUGCGGCUCA BMPR2 3'UTR mut AGGUUGUUGCAGUGACGGCTCA

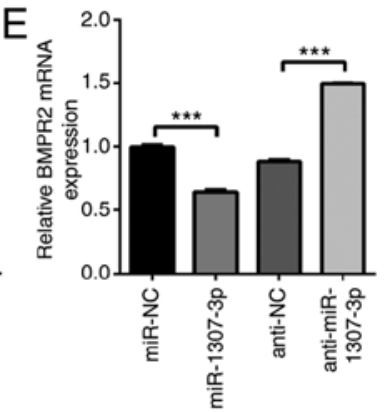

$\mathrm{F}$
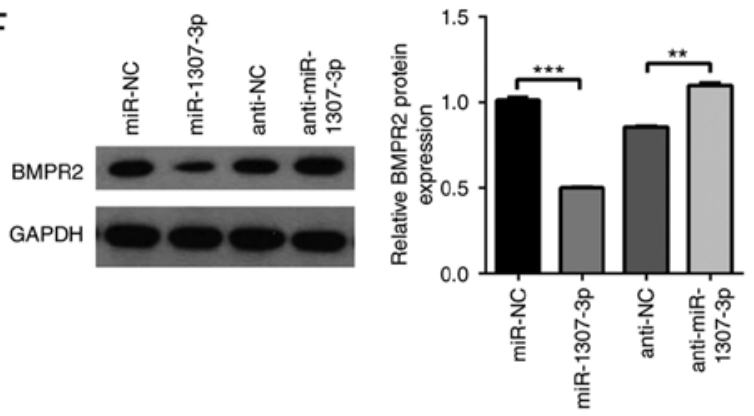

Figure 3. BMPR2 is a direct target of miR-1307-3p. The expression of BMPR2 during chondrogenic differentiation was examined by (A) RT-qPCR and (B) western blot analyses. (C) Predicted binding site of miR-1307-3p in the 3'UTR of BMPR2 is shown. (D) hADSCs were transfected with the indicated plasmids, and the luciferase activities were determined using a dual luciferase assay. hADSCs were infected with the indicated recombinant lentivirus, and (E) mRNA and (F) protein levels of BMPR2 were analyzed by RT-qPCR and western blot analyses, respectively. ${ }^{*} \mathrm{P}<0.05,{ }^{* * *} \mathrm{P}<0.01$ and ${ }^{* * * *} \mathrm{P}<0.001$. miR, microRNA; hADSCs, human adipose-derived stem cells; BMPR2, bone morphogenetic protein receptor type 2; 3'UTR, 3' untranslated region; wt, wild-type; mut, mutant; NC, negative control; RT-qPCR, reverse transcription-quantitative polymerase chain reaction; NS, not significant; d, day.
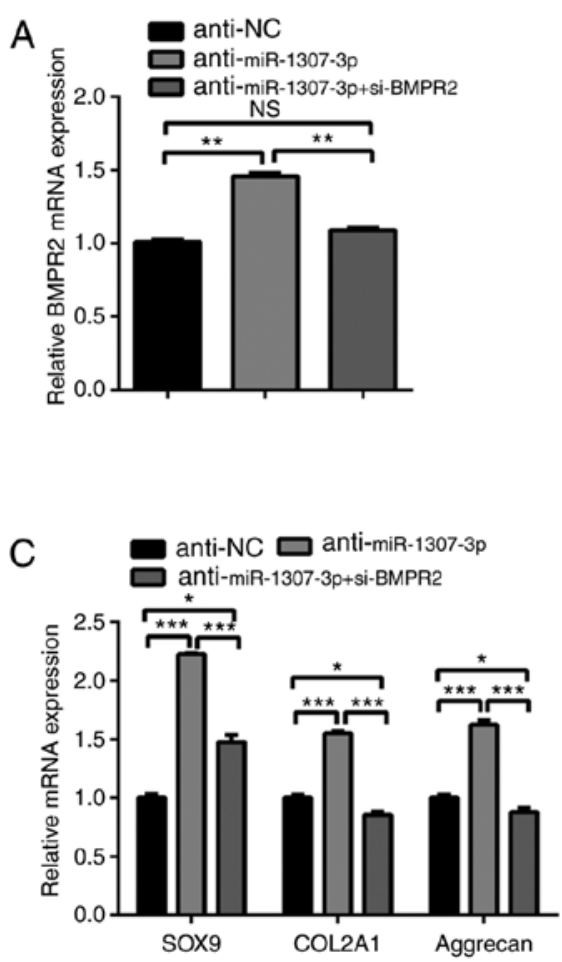

B
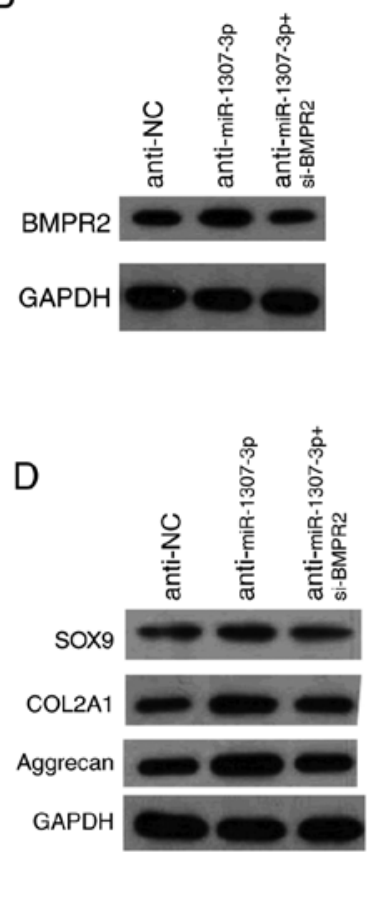
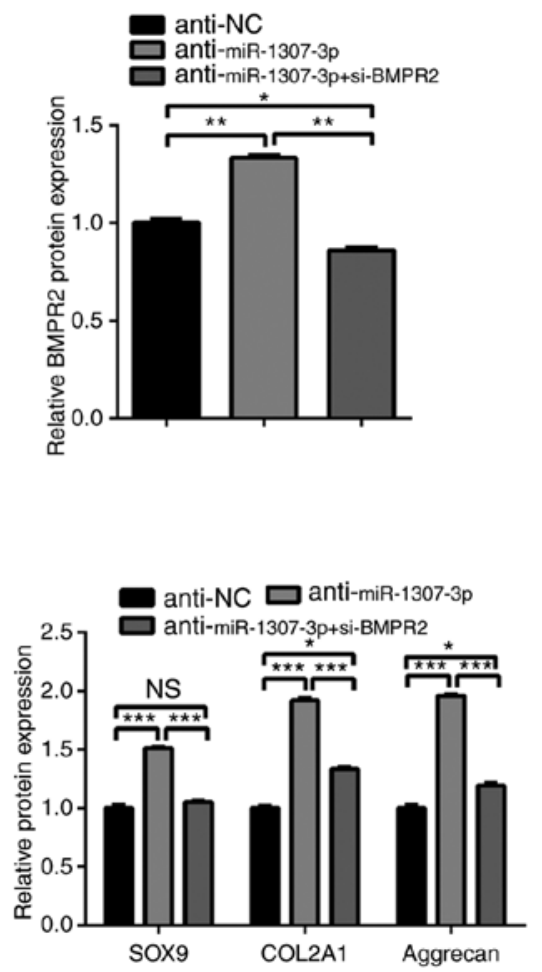

Figure 4. BMPR 2 mediates the inhibitory effect of miR-1307-3p on the chondrogenic differentiation of hADSCs. hADSCs were infected or transfected with the indicated lentivirus or siRNA. (A) mRNA and (B) protein levels of BMPR2 were determined by RT-qPCR and western blot analyses, respectively. (C) mRNA and (D) protein levels of SOX9, COL2A1 and Aggrecan were determined by RT-qPCR and western blot analyses, respectively. ${ }^{*} \mathrm{P}<0.05$, ${ }^{* *} \mathrm{P}<0.01$ and ${ }^{* * *} \mathrm{P}<0.001$. miR, microRNA; hADSCs, human adipose-derived stem cells; BMPR2, bone morphogenetic protein receptor type 2; si-, small interfering RNA; SOX9, sex determining region Y-box 9; COL2A1, collagen type II $\alpha 1$ chain; NC, negative control; RT-qPCR, reverse transcription-quantitative polymerase chain reaction; NS, not significant.

proteins was markedly decreased when miR-1307-3p was overexpressed, whereas their phosphorylation was increased when miR-1307-3p was knocked down (Fig. 5A and B). Furthermore, BMPR2 knockdown prevented the promoting 

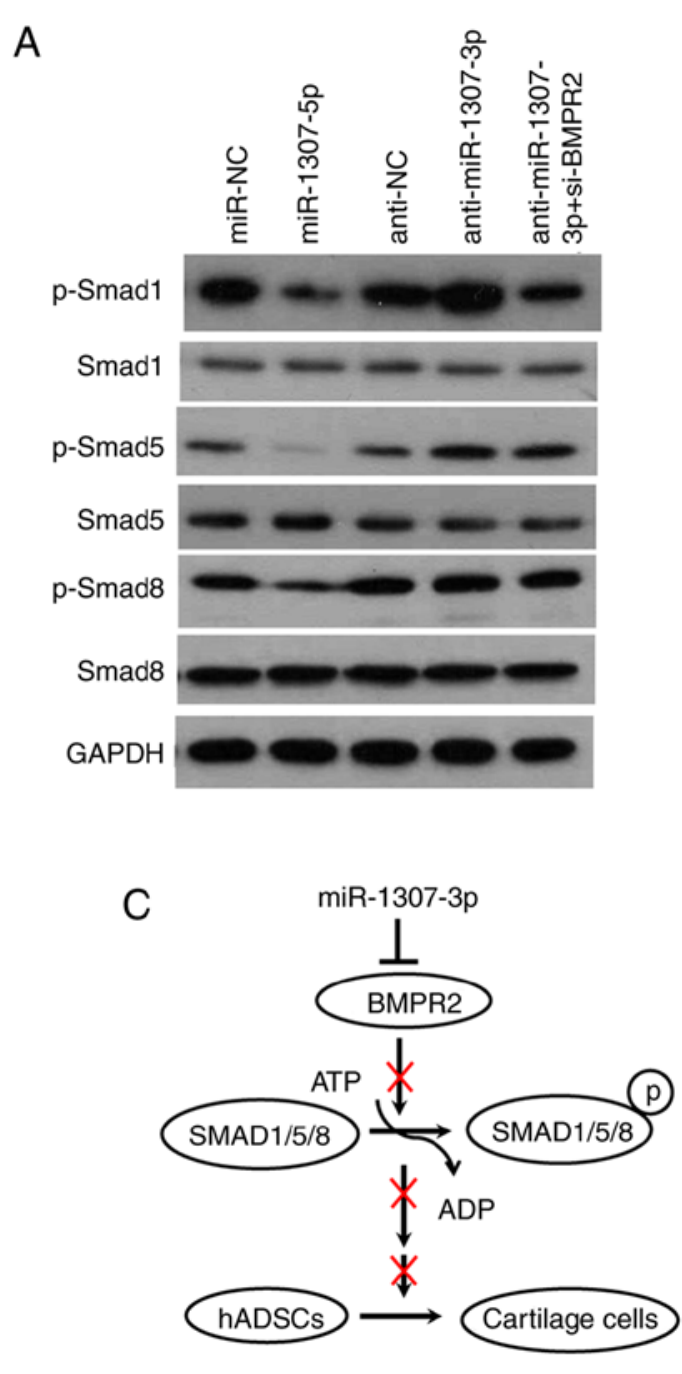

$\mathrm{B}$
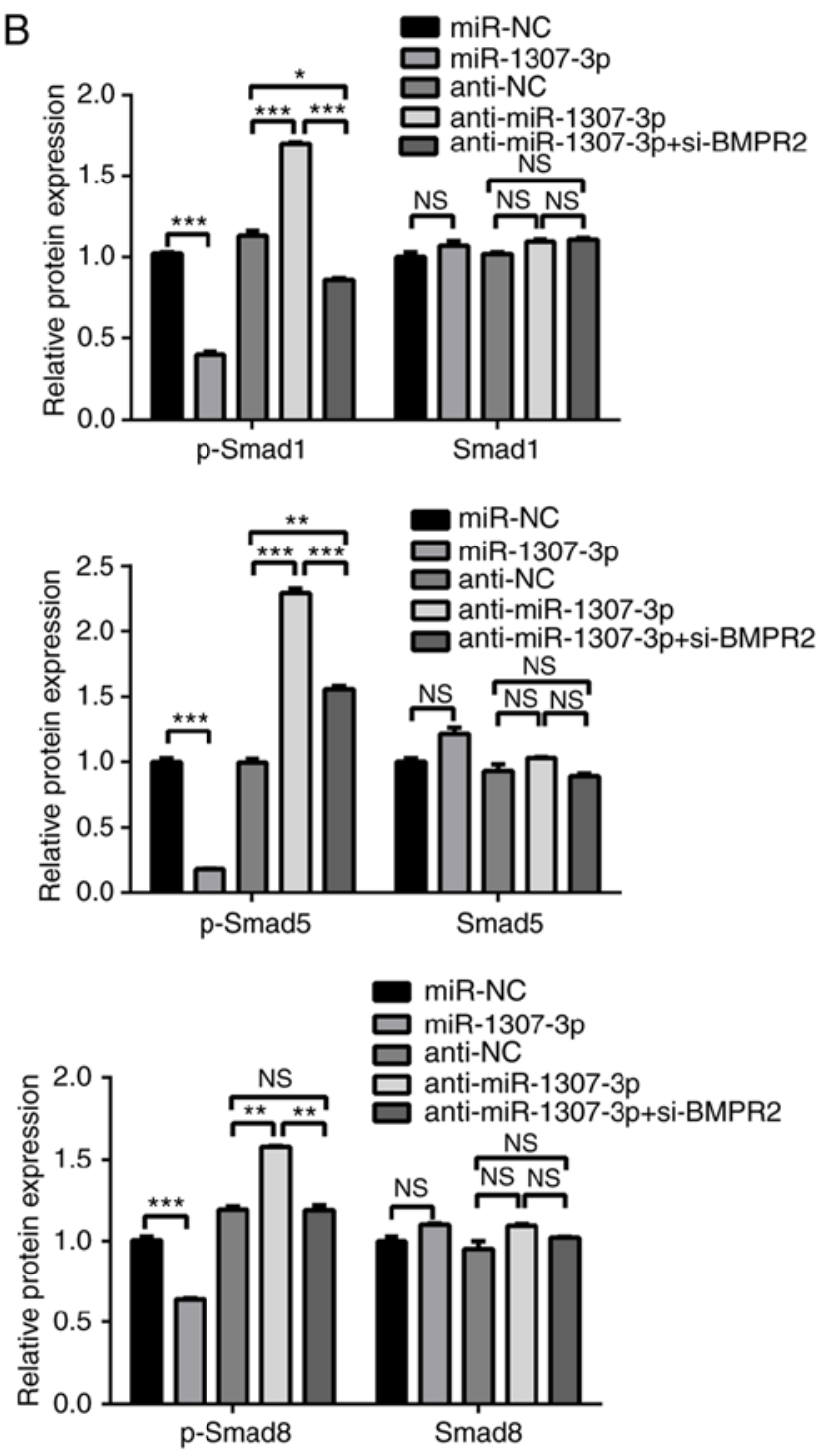

Figure 5. miR-1307-3p inhibits the BMPR2-SMAD pathway. (A) hADSCs were infected with the indicated recombinant lentivirus, and the protein levels of Smad1, 5, and 8, and the phosphorylation of Smad1, 5, and 8 were analyzed by western blot analysis. (B) Graphs show quantification of western blot results relative to $\beta$-actin. (C) Proposed model for the suppression of miR-1307-3p on chondrogenic differentiation. ${ }^{*} \mathrm{P}<0.05,{ }^{* *} \mathrm{P}<0.01$ and ${ }^{* * * *} \mathrm{P}<0.001$. miR, microRNA; hADSCs, human adipose-derived stem cells; BMPR2, bone morphogenetic protein receptor type 2; si-, small interfering RNA; SMAD, mothers against decapentaplegic; p-, phosphorylated; NC, negative cotrol; NS, not significant.

effect of miR-1307-3p inhibition on the phosphorylation of the three Smad proteins (Fig. 5A and B). These results suggested that miR-1307-3p suppressed chondrogenic differentiation via inhibiting BMPR2 downstream pathways.

\section{Discussion}

Emerging data has shown that miRNAs may exert key functions in the chondrogenic differentiation of hADSCs (9). According to the miRNA microarray and northern blot analysis in our previous study, miR-1307-3p was significantly downregulated during the chondrogenic differentiation of hADSCs (13). In the present study, its decreased expression was verified with RT-qPCR analysis. Previous data show that it may be used as a valuable marker for the diagnosis and treatment of certain diseases, however, the function of miR-1307-3p has not been determined (19-22). Johnson et al (19) found that the combinations of five miRNAs, including miR-1307-3p, in the saliva of children can identify patients with prolonged symptoms on a logistic regression. The polymorphism rs7911488 $\mathrm{T}>\mathrm{C}$ in pre-miR-1307p was closely associated with the efficacy of capecitabine chemotherapy in patients with colon cancer (20). García-Donas et al (21) found that the expression of miR-1307-03p was increased in tumor tissues from cases of metastatic renal cell carcinoma uniformly treated with tyrosine kinase inhibitors by deep sequencing of miRNA expression profiles. Its expression was significantly associated with progression-free survival and overall survival rates, which suggest it may be a useful biomarker for metastatic renal cell carcinoma treatment. Shimomura et al (22) found that the level of miR-1307 was increased in breast cancer, and its combination with the four other miRNAs was a useful marker for the diagnosis of breast cancer. In the present study, the results demonstrated that miR-1307-3p suppressed the chondrogenic differentiation of hADSCs by targeting BMPR 2 and inhibited the BMPR2 downstream signaling pathway. 
miRNAs function at post-transcriptional levels by degrading target mRNAs or inhibiting the translation of target mRNAs. To identify targets of miR-1307-3p, the present study used two online software tools, TargetScan and PicTar, to predict its targets and found that BMPR2 is a potential target of miR-1307-3p. Its expression during the chondrogenic differentiation of hADSCs was increased, which was negatively correlated with the decreased levels of miR-1307-3p. A luciferase assay showed that BMPR2 was the target of miR-1307-3p. RT-qPCR and western blot analyses showed that miR-1307-3p suppressed the endogenous expression of BMPR2. The rescue experiments indicated that the inhibitory function of miR-1307-3p on the chondrogenic differentiation of hADSCs was mediated by BMPR2. As miRNAs can often target more than one target, whether other targets are involved in the effect of miR-1307-3p on the chondrogenic differentiation of hADSCs requires further investigation.

BMPR2, a member of the BMP receptor family of transmembrane serine/threonine kinases, is involved in the differentiation of mesenchymal progenitor cells (23). It promotes osteogenic or chondrogenic differentiation, and can be targeted and negatively regulated by miR-99a, miR-153, miR-490 and miR-100, respectively (13,24-26). BMPR2 is a key molecule in BMP signaling. On binding to BMP, BMPR2 is phosphorylated and activates BMPR1, which in turn leads to the phosphorylation of intracellular Smad1, 5, and 8. Subsequently, the common mediator Smad4 binds to phosphorylated Smad1, 5, and 8 and is translated into the nucleus, which can activate the transcription of BMPR target genes $(27,28)$. In the present study, it was found that miR-1307-3p suppressed the chondrogenic differentiation of hADSCs by targeting and inhibiting the expression of BMPR2. To further examine the specific mechanism, the expression of molecules of the BMP downstream signaling pathway was assessed. It was found that the overexpression of miR-1307-3p decreased the phosphorylation of Smad1, 5, and 8 , whereas the inhibition of miR-1307-3p had the opposite effects. However, the knockdown of BMPR2 attenuated the increased phosphorylation of Smad1, 5, and 8 by miR-1307-3p. These results indicated that the suppression of chondrogenic differentiation by miR-1307-3p was due to the inhibition of the BMPR2-Smad signaling pathway.

In conclusion, the results of the present study demonstrated that miR-1307-3p suppressed the chondrogenic differentiation of hADSCs by targeting BMPR 2 and subsequently inhibiting the BMPR2-Smad signaling pathway. These findings assist in understanding the molecular mechanisms underlying chondrogenic differentiation, which may provide novel therapeutic approaches for hADSC-based cartilage tissue engineering.

\section{Acknowledgements}

Not applicable.

\section{Funding}

This study was supported by the Youth Fund of Guizhou Provincial People's Hospital [grant no. GZSYQN(2015)06], the National Natural Science Foundation of China (grant nos. 31660265, 81060145 and 81560356), the Subsidy
Foundation of National Natural Science Foundation of Guizhou Provincial People's Hospital [Guizhou Science and Technology Platform (grant no. (2017)5724], and the Science and Technology Foundation of Guizhou Province [Guizhou Science and Technology J Word (2015)2096].

\section{Availability of data and materials}

All data generated or analyzed during this study are included in this published article.

\section{Authors' contributions}

$\mathrm{BL}$ and $\mathrm{XBT}$ were involved in the study design and revision of the manuscript. ZY and RL wrote the manuscript. ZY, RL, JA, QDW and YZ performed the experiments. ZY, LC, JW, BC and WP analysed and interpreted the data. All authors read and approved the final manuscript.

\section{Ethics approval and consent to participate}

The study was approved by the Ethics Committee of Tianjin Haihe Hospital (Tianjin, China). Written informed consent was obtained from the donors.

\section{Patient consent for publication}

Written informed consent was obtained from the donors.

\section{Competing interests}

The authors declare that they have no competing interests.

\section{References}

1. Ge Z, Hu Y, Heng BC, Yang Z, Ouyang H, Lee EH and Cao T: Osteoarthritis and therapy. Arthritis Rheum 55: 493-500, 2006.

2. Zhang $\mathrm{J}$ and Chen $\mathrm{J}$ : Bone tissue regeneration-Application of mesenchymal stem cells and cellular and molecular mechanisms. Curr Stem Cell Res Ther 12: 357-364, 2017.

3. Vallée M, Côté JF and Fradette J: Adipose-tissue engineering: Taking advantage of the properties of human adipose-derived stem/stromal cells. Pathol Biol (Paris) 57: 309-317, 2009.

4. Wang S, Qu X and Zhao RC: Mesenchymal stem cells hold promise for regenerative medicine. Front Med 5: 372-378, 2011.

5. Zuk PA, Zhu M, Ashjian P, De Ugarte DA, Huang JI, Mizuno H, Alfonso ZC, Fraser JK, Benhaim P and Hedrick MH: Human adipose tissue is a source of multipotent stem cells. Mol Biol Cell 13: 4279-4295, 2002.

6. Gimble J and Guilak F: Adipose-derived adult stem cells: Isolation, characterization, and differentiation potential. Cytotherapy 5: 362-369, 2003.

7. Bartel DP: MicroRNAs: Genomics, biogenesis, mechanism, and function. Cell 116: 281-297, 2004.

8. Dong H, Lei J, Ding L, Wen Y, Ju H and Zhang X: MicroRNA: Function, detection, and bioanalysis. Chem Rev 113: 6207-6233, 2013.

9. Wu C, Tian B, Qu X, Liu F, Tang T, Qin A, Zhu Z and Dai K: MicroRNAs play a role in chondrogenesis and osteoarthritis (Review). Int J Mol Med Jul 34: 13-23, 2014.

10. Lee S, Yoon DS, Paik S, Lee KM, Jang Y and Lee JW: microRNA-495 inhibits chondrogenic differentiation in human mesenchymal stem cells by targeting Sox9. Stem Cells Dev 23: 1798-1808, 2014.

11. Zhang Y, Huang X and Yuan Y: MicroRNA-410 promotes chondrogenic differentiation of human bone marrow mesenchymal stem cells through down-regulating Wnt3a. Am J Transl Res 9: 136-145, 2017. 
12. Zhang Z, Kang Y, Zhang Z, Zhang H, Duan X, Liu J, Li X and Liao W: Expression of microRNAs during chondrogenesis of human adipose-derived stem cells. Osteoarthritis Cartilage 20: 1638-1646, 2012.

13. Yang Z, Hao J and Hu ZM: MicroRNA expression profiles in human adipose-derived stem cells during chondrogenic differentiation. Int J Mol Med 35: 579-586, 2015.

14. Hou C, Yang Z, Kang Y, Zhang Z, Fu M, He A, Zhang Z and Liao W: MiR-193b regulates early chondrogenesis by inhibiting the TGF-beta2 signaling pathway. FEBS Lett 589: 1040-1047, 2015.

15. Hou C, Zhang Z, Zhang Z, Wu P, Zhao X, Fu M, Sheng P, Kang Y and Liao W: Presence and function of microRNA-92a in chondrogenic ATDC5 and adipose-derived mesenchymal stem cells. Mol Med Rep 12: 4877-4886, 2015.

16. Xu J, Kang Y, Liao WM and Yu L: MiR-194 regulates chondrogenic differentiation of human adipose-derived stem cells by targeting Sox 5. PLoS One 7: e31861, 2012.

17. Wu SC, Hsiao HF, Ho ML, Hung YL, Chang JK, Wang GJ and Wang CZ: Suppression of discoidin domain receptor 1 expression enhances the chondrogenesis of adipose-derived stem cells. Am J Physiol Cell Physiol 308: C685-C896, 2015.

18. Livak KJ and Schmittgen TD: Analysis of relative gene expression data using real-time quantitative PCR and the 2- $\Delta \Delta C \mathrm{CT}$ method. Methods 25: 402-408, 2001.

19. Johnson JJ, Loeffert AC, Stokes J, Olympia RP, Bramley H and Hicks SD: Association of salivary microRNA changes with prolonged concussion symptoms. JAMA Pediatr 172: 65-73, 2018.

20. Chen Q, Mao Y, Meng F, Wang L, Zhang H, Wang W and Hua D: Rs7911488 modified the efficacy of capecitabine-based therapy in colon cancer through altering miR-1307-3p and TYMS expression. Oncotarget 8: 74312-74319, 2017.

21. García-Donas J, Beuselinck B, Inglada-Pérez L, Graña O, Schöffski P, Wozniak A, Bechter O, Apellániz-Ruiz M, Leandro-García LJ, Esteban E, et al: Deep sequencing reveals microRNAs predictive of antiangiogenic drug response. JCI Insight 1: e86051, 2016.
22. Shimomura A, Shiino S, Kawauchi J, Takizawa S, Sakamoto H, Matsuzaki J, Ono M, Takeshita F, Niida S, Shimizu C, et al: Novel combination of serum microRNA for detecting breast cancer in the early stage. Cancer Sci 107: 326-334, 2016.

23. Kang Q, Song WX, Luo Q, Tang N, Luo J, Luo X, Chen J, Bi Y, He BC, Park JK, et al: A comprehensive analysis of the dual roles of BMPs in regulating adipogenic andosteogenic differentiation of mesenchymal progenitor cells. Stem Cells Dev 18: 545-559, 2009.

24. Zhou X, Wang J, Sun H, Qi Y, Xu W, Luo D, Jin X, Li C, Chen W, Lin Z, et al: MicroRNA-99a regulates early chondrogenic differentiation of rat mesenchymal stem cells by targeting the BMPR2 gene. Cell Tissue Res 366: 143-53, 2016.

25. Cao Y, LV Q and LV C: MicroRNA-153 suppresses the osteogenic differentiation of human mesenchymal stem cells by targeting bone morphogenetic protein receptor type II. Int J Mol Med 36: 760-766, 2015.

26. Zeng Y, Qu X, Li H, Huang S, Wang S, Xu Q, Lin R, Han Q, Li J and Zhao RC: MicroRNA-100 regulates osteogenic differentiation of human adipose-derived mesenchymal stem cells by targeting BMPR2. FEBS Lett 586: 2375-2381, 2012.

27. Liu F, Ventura F, Doody J and Massagué J: Human type II receptor for bone morphogenic proteins (BMPs): Extension of the two-kinase receptor model to the BMPs. Mol Cell Bio 15: 3479-3486, 1995.

28. Miyazono K, Maeda S and Imamura T: BMP receptor signaling: transcriptional targets, regulation of signals, and signaling cross-talk. Cytokine Growth Factor Rev 16: 251-263, 2005.

This work is licensed under a Creative Commons Attribution-NonCommercial-NoDerivatives 4.0 International (CC BY-NC-ND 4.0) License. 